\title{
Erratum to: Minimally invasive lateral transpsoas approach with advanced neurophysiologic monitoring for lumbar interbody fusion
}

Pedro Berjano • Claudio Lamartina

Published online: 19 October 2011

(C) Springer-Verlag 2011

Erratum to: Eur Spine J (2011) 20:1584-1586

DOI 10.1007/s00586-011-1997-x

The following affiliation was omitted:

I.R.C.C.S Istituto Ortopedico Galeazzi, Milan, Italy.

e-mail: c.lamartina@chirurgiavertebrale.net.

The online version of the original article can be found under doi:10.1007/s00586-011-1997-x.

P. Berjano · C. Lamartina $(\bowtie)$

I.R.C.C.S Istituto Ortopedico Galeazzi, Milan, Italy

e-mail: c.lamartina@chirurgiavertebrale.net 